
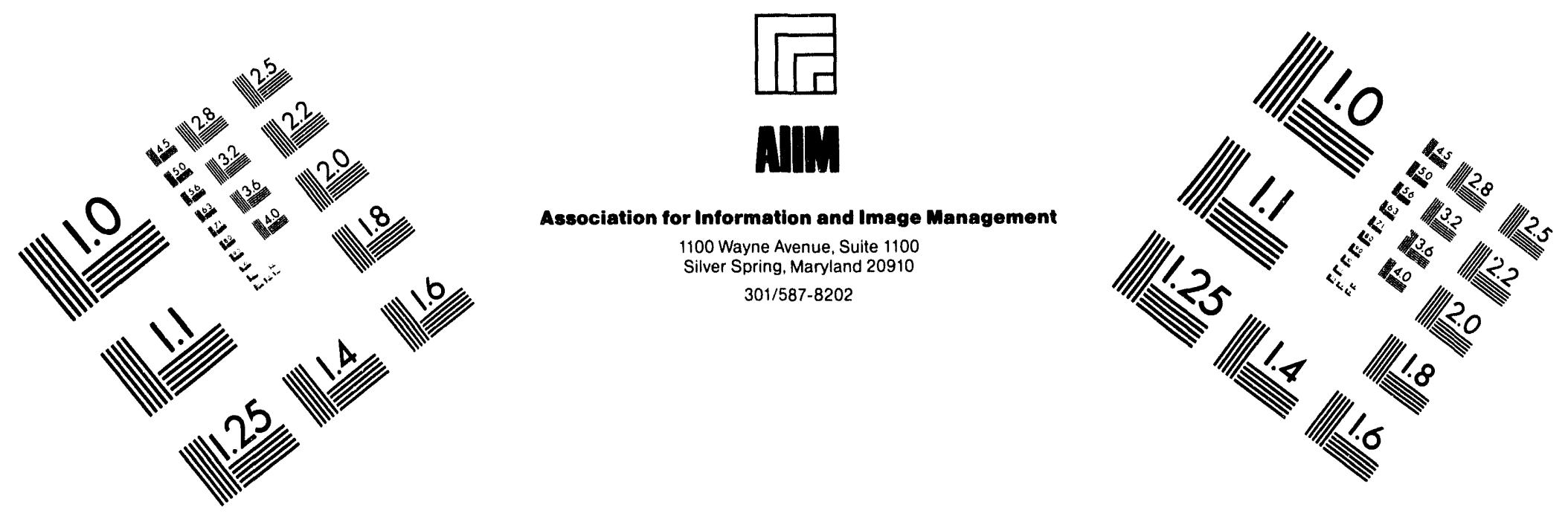

\title{
Centimeter
}

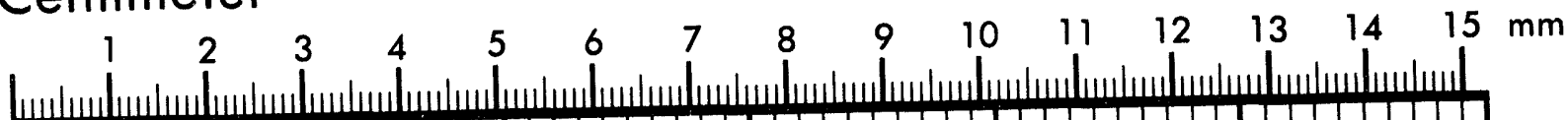
$\mid$ Inches
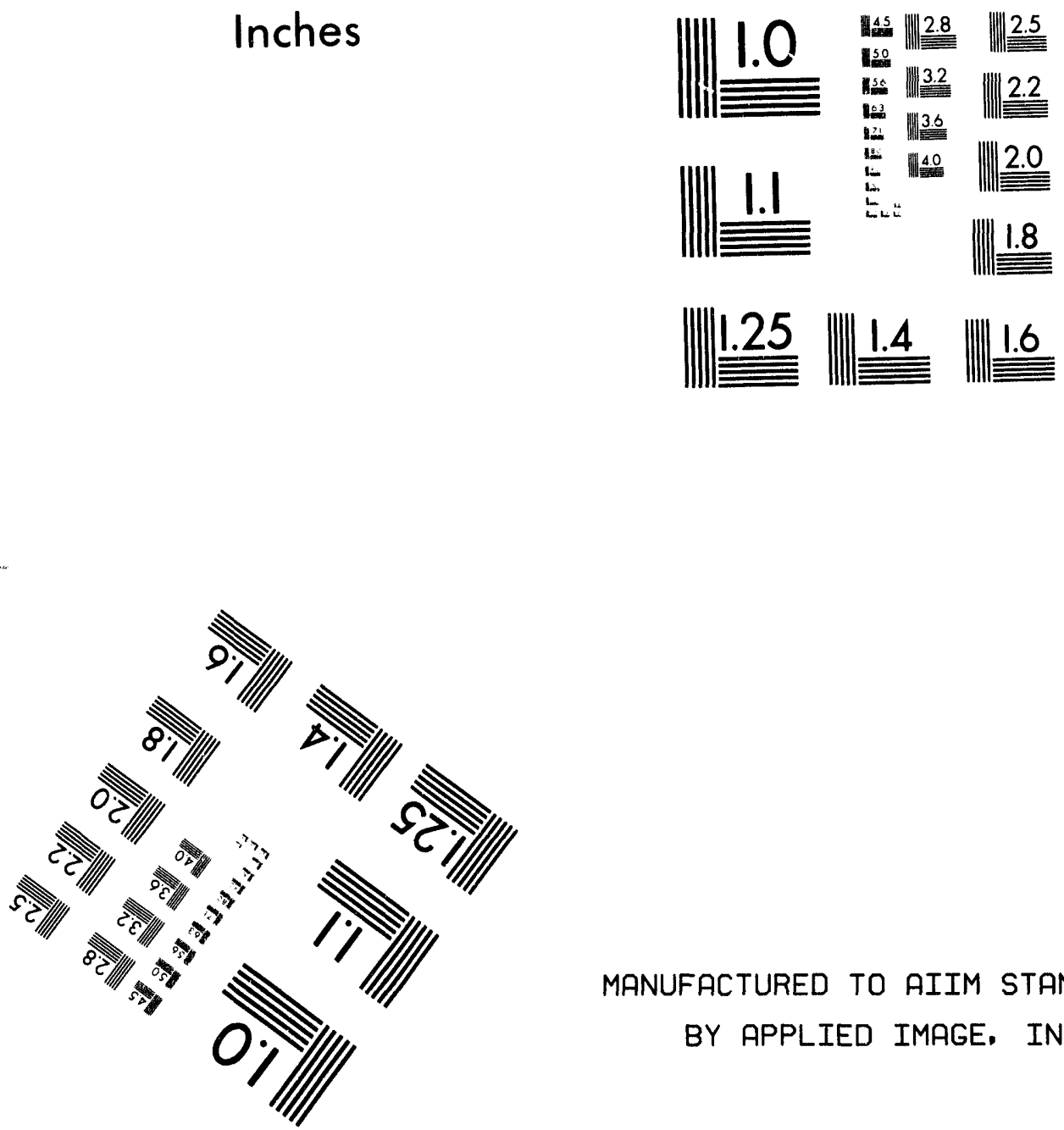

MANUFACTURED TO AIIM STANDARDS

BY APPLIED IMAGE. INC.

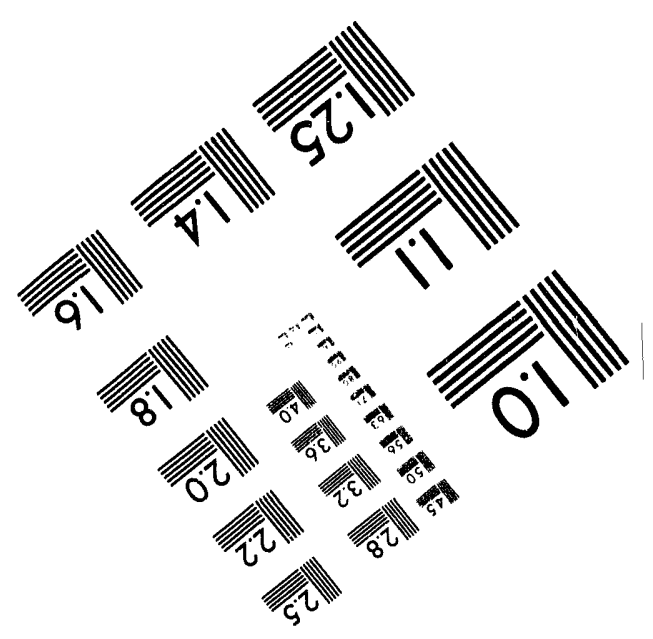



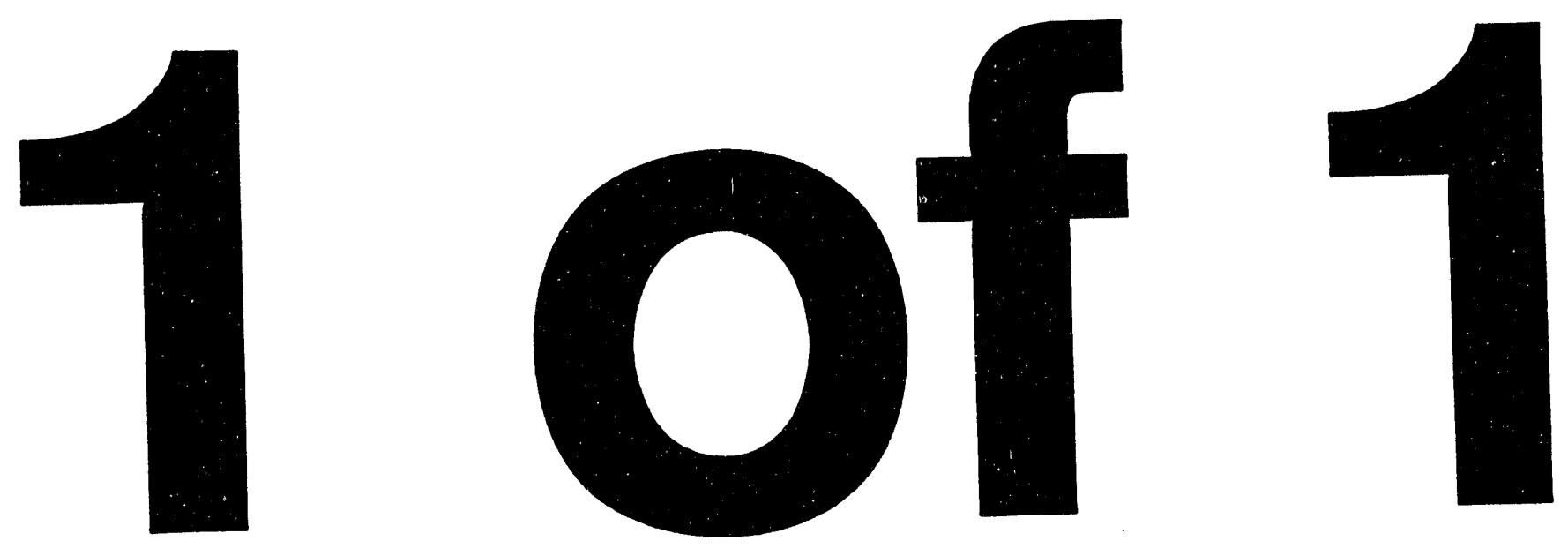


\title{
SAND- $-94-8701 C$ Conf-940838--?
}

Topical Review for 25th Combustion Symposium - draft May 10, 1994

\section{Modeling Turbulent Flame Propagation}

\author{
Wm. T. Ashurst \\ Combustion Research Facility \\ Sandia National Laboratories \\ Livermore, CA 94551-0969 USA
}

\begin{abstract}
Laser diagnostics and flow simulation techniques are now providing information that, if available fifty years ago, would have allowed Damköhler to show how turbulence generates flame area. In the absence of this information, many turbulent flame speed models have been created, most based on Kolmogorov concepts which ignore the turbulence vortical structure. Over the last twenty years, the vorticity structure in mixing layers and jets has been shown to determine the entrainment and mixing behavior and these effects need to be duplicated by combustion models. Turbulence simulations reveal the intense vorticity structure as filaments and simulations of passive flamelet propagation show how this vorticity creates flame area and defines the shape of the expected chemical reaction surface. Understanding how volume expansion interacts with flow structure should improve experimental methods for determining turbulent flame speed. Since the last decade has given us such powerful new tools to create and see turbulent combustion microscopic behavior, it seems that a.solution of turbulent combustion within the next decade would not be surprising in the hindsight of 2004 .
\end{abstract}

\section{DISCLAIMER}

This report was prepared as an account of work sponsored by an agency of the United States Government. Neither the United States Government nor any agency thereof, nor any of their employees, makes any warranty, express or implied, or assumes any legal liability or responsibility for the accuracy, completeness, or usefulness of any information, apparatus, product, or process disclosed, or represents that its use would not infringe privately owned rights. Reference herein to any specific commercial product, process, or service by trade name, trademark, manufacturer, or otherwise does not necessarily constitute or imply its endorsement, recommendation, or favoring by the United States Government or any agency thereof. The views and opinions of authors expressed herein do not necessarily state or reflect those of the United States Government or any agency thereof. 


\section{Introduction}

Forman Williams, in the last Hottel Lecture, described the role of theory in combustion science. He excluded modeling and simulation based on computation and this is the theme of our review. One area of combustion modeling that has grown since Williams' lecture [1] and the book with Liñán [2] is the Linear Eddy work by Kerstein and co-workers. This model uses random stirring with a deterministic structure in scalar diffusion and reaction, but details of this work will be left to their Symposium papers. Also, the current Hottel lecture by Frank Marble on Gasdynamic Enhancement of Nonpremixed Combustion provides another reason for ignoring nonpremixed combustion in this review.

Therefore, which new aspects of combustion science should concern modeling premixed combustion? My favorite is the structure of turbulence.' Numerical simulations of turbulence have revealed an intense vorticity structure that is tube-like at low Mach number and sheet-like at larger flow speeds [3]. How will a premixed flame-move through this structure? Since the simulation of turbulence and combustion combined is too much for computers of today, we look for ways to separate these two problems, and focus the computer power on just one aspect. From personal experience, my viewpoint is that vorticity and its structure are vital to entrainment and molecular mixing and therefore vital to combustion models. In jets and mixing layers, these moving structures produce a smooth time-average Eulerian velocity field which does not resemble the Lagrangian flow pattern around the structure. The important point is that flame motion occurs in the Lagrangian frame and the historical difficulty is that experimental information was acquired in the Eulerian frame. Grasping how these views differ is now easier with simulation techniques and the global information given by laser sheet techniques. When viewed in its reference frame, the local swirling motion around a vortex is a simple flow pattern; the experimental difficulty is finding that reference frame.

In this review, we give a brief description of flow vorticity structure and a more detailed presentation of computed turbulence structure. We then discuss flamelet models which are used to decouple chemistry from turbulence. The propagation of flamelets through this structure has provided concepts for modoling turbulent, spherically expanding flames and steady planar flames. A recent example of detailed chemistry with simulated two-dimensional tiurbulence shows what will become common with the larger and faster computers of tomorrow. 


\section{Flow Vorticity Structure}

It has been twenty years since the Brown \& Roshko publication on the vortical structure of a mixing layer [4]. In these layers, the Kelvin-Helmholtz instability creates spanwise vortical rollers and these structures pair together and form larger structures, this process continues as long as the outer flow conditions maintain a velocity difference across the layer. Between these rollers there appears counter-rotating vortex pairs orientated in the streamwise direction. Pairs of opposite rotation will form if the spanwise vorticity is pulled into the flow direction, acquiring a horseshoe shape. A straight vortex tube has a symmetric rotation about its axis and therefore, no net flux in any plane containing the axis. However, a counter-rotating pair, similar to a vortex loop, will create a net flux of momentum and scalar normal to the plane of the loop. The loop shape may not remain in the initial plane because the self-induced velocity depends on the curvature magnitude and this will form curved vortex sections in planes normal to the initial plane. 'The topology may also change via a reconnection process [5], a common sight are the loops formed in the contrails behind a high-altitude jet - these loops often form a régular structure with downward motion.

Current understanding [6] of the mixing behavior in these flows is that the second vortex pairing creates a mixing transition (the streamwise vortical structure has formed) and at this flow location there is a scalar pdf which does not change shape with location across the layer; the third vortex pairing creates a broad-marching pdf, that is the peak value changes with location. The large-scale structures affect the overall mixing process and the number of pairings, rather than Reynolds number by itself, characterize the mixing behavior. From direct numerical simulations of a mixing layer, Lopez \& Bulbeck [7] have examined the vorticity structure in the streamwise direction and find the Burgers' vortex is a valid solution where the axial strain is extensional, but where it is compressional the vorticity spirals opposite its own swirl direction. This vortex breakdown mechanism may set the length scale of the vortex.

The streamwise vorticity structure in a jet has been determined by Liepmann \& Gharib [8] using the DPIV technique, they find these structures drastically alter the entrainment process and become more dominant than the ring mode of vorticity; simulations are given in [9]. Jets with a non-round orifice have been used to enhance entrainment via the deformation of the vortex structures, Hertzberg \& Ho [10] have examined a rectangular jet in which the downstream wall confinement is a constant distance from the orifice perimeter. 
Freely expanding non-round jets exhibit a larger spreading in the minor axis plane, in this confined flow the three-dimensional effects combined with this spreading creates no back flow in the minor axis plane. Understanding and then controlling these three-dimensional structures offers a way to control mixing and hence combustion. Jets with axial forcing controlling the lifted flame behavior are in [11] and additional azimuthal perturbations are in the study by Lasheras et al. [12]. Details of a swirling jet flow are given next [13].

Swirling flows are often used to promote mixing with controlled residence times, yet the analysis of the instantaneous flow structure has not developed to the point that even mean spreading angle of the flow can be predicted. While some success has been achieved with a second-order closure of the Reynolds-averaged Navier-Stokes equations, there is concern regarding the use of the Reynolds' decomposition in this swirling flow, especially when dispersion of spray droplets and the resulting unsteady evaporation must be included for combustion applications.

In this swirl flow there is a centerbody, which houses the spray mechanism, and so the mean axial flow is annular jet with reverse flow along the centerline near the exit plane. All components of the mean velocity indicate an axisymmetric pattern, with large fluctuation values compared to the mean values. However, instantaneous images of the swirling jet fluid do not resemble an axisymmetric pattern, but indicate a Kelvin-Helmholtz roll-up on the outer edge which is not symmetric with respect to the centerline. These outer structures form an eddy of viry large scale, and this eddy moves downstream along a path with increasing radius. Images taken normal to the axial flow give the impression of a helical structure, a left-handed helix with a pitch corresponding to the jet diameter; the flow swirl is right-handed, see Figure 1. Thus, the instantaneous flow pattern does not resemble the time-average mean flow and so the motion of drops as calculated from the Reynolds-decomposition may not resemble the actual drop motion. In particular, measurements indicate regions where the average radial velocity is outward, as are drops above a certain size ( $\sim 20$ microns), but smaller drops are moving inward at this location! The formation of these structures creates a fluctuation velocity that is larger than the mean velocity, and so in this spatial region the mean velocity no longer has much meaning. 


\section{Vorticity Structure in Turbulence}

The equations of motion were developed by Newton in 1686 and those for a viscous fluid by Navier in 1822 and Stokes in 1845. Just one hundred years ago this Spring, Reynolds suggested a decomposition into a mean velocity and the temporal fluctuation about that mean to describe turbulence. Taylor, in 1935, developed a statistical approach. In 1941, Kolmogorov assumed the velocity difference between two points separated by the distance $r$ would depend on that distance, the viscosity and the energy dissipation in the turbulent flow. When the distance grew large the effect of viscosity would disappear leaving only the energy dissipation to characterize a turbulent flow. This implies (though not contained within the 1941 paper) the $-5 / 3$ power law wavenumber dependence of the energy spectrum.

The experimental techniques, and thereby the conceptual view, promoted the Fourier description of the flow and hence the energy spectrum was the focus. The physical flow pattern was dismissed as too complicated, it was turbulent, and so the phase angle of the Fourier modes was also too much to deal with. Only with the decade of the 80 's were computers and computer graphics advanced enough so that simulated turbulence and the fiow pattern could be easily examined. The critical importance of phase angle is shown by She et al. [14] in which the computed flow vorticity is imaged along with "vorticity" from the same Fourier modes but with random phase angles. The two flows have exactly the same spectrum but do not look alike in physical space. For combustion models, which need entrainment and molecular mixing, the physical flow pattern is of vital concern, and not just any phase angle with a given energy spectrum will do.

The structure of vorticity and its role in stability has recently been recognized as being more essential than previously assumed. A significant concept change is the use of three-dimensional, nonmodal fluid motion which leads to finite amplitude deformation via interactions between eigenfunctions which are not orthogonal to one another. The review by Trefethen et al. [15] describes the effect of a streamwise vortex in a shear flow, in which the fluid motion amplifies in an inviscid manner before the effect of viscosity occurs. "Such behavior is physically straightforward, appearing complicated only when interpreted in the basis of eigenmodes."

\section{Status of the Largest Turbulence Simulations}

Using a parallel computer (CM-2 at Los Alamos National Laboratory), simulations of forced and decaying turbulence have been performed with a grid mesh of $512^{3}$, the largest 
Reynolds number is $R e_{\lambda} \sim 200$, see Chen et al. [16] and She et al. [17]. The focus of these large simulations is understanding the intermittent turbulence structure. Kolmogorov added a third hypothesis in 1962 to account for fluctuations in the energy dissipation, but until recently, this intermittency effect eluded theory and experimentation. Their $512^{3}$ results are: 1$)$ energy spectra scaled by $k_{p}$ and $E\left(k_{p}\right)$ collapse and agree with experimental results at much higher Reynolds number; 2) variation of $k_{p}$, which is the peak wave number in the dissipation spectrum, is consistent with the 1941 Kolmogorov theory in that $k_{p}$ has a linear relation to $k_{d}$, the Kolmogorov wave number $\left(k_{d}{ }^{4}=\epsilon / \nu^{3}\right)$; and 3) velocity incrernent results indicate intermittency growth with decrease in distance. Given results 1 and 2 in contrast to 3, why is the Kolmogorov theory so good for the energy spectrum but not for the higher powers of the velocity increments? She [17] suggests that structures which dominate intermittency do not have a large contribution to the mean transfer of energy. However, the swirling flow about these structures creates flame area and curvature, and for this reason, we concentrate on their features in this review.

\section{Models of Turbulence Structure}

Experimental measurement of higher powers of the velocity difference do not agree with the 1941 theory which gives a spatial scaling with exponent of $p / 3$ for the power $p$ of the velocity difference, or increment. She [18] has used the filament shape of the intense vorticity, revealed by direct numerical simulations of turbulence, in order to develop a universal scaling of the velocity increments without adjustable parameters. There are two assumptions: 1) successive moments of the energy dissipation averaged over a volume of size $l$ have a universal relation to the infinite moment of the dissipation, and 2) the structures that give the most intermittent events are filaments of vorticity. The filamentary nature sets a codimension in the model and the resulting exponents of the velocity increment to the power $p$ are $\zeta_{p}=p / 9+2\left[1-(2 / 3)^{p / 3}\right]$. The correction to the energy spectrum with this scaling model is small $\approx k^{-(5 / 3)-0.03}$, but the departure from higher exponents of the Kolmogorov theory is large, and this new scaling gives values of $\zeta_{p}$ which are within experimental uncertainty, for example, the tenth power model result is 2.593 , the experimental value is 2.60 and the Kolmogorov is 3.33 .

\section{Computed Vortical Structure}

Jiménez et al. [19] have performed direct numerical simulations with four grid sizes, $64^{3}$ to $512^{3}$, with $35<R e_{\lambda}<170$, where the Reynolds number is based on the Taylor scale 
$\lambda$. These simulations are at constant dissipation, the forcing used a negative viscosity in the lowest wavenumbers so that $K \eta \approx 2$, where $K$ is the largest resolved wavenumber. The dissipation is determined from the energy spectrum and this in turn defines the Kolmogorov length as $\epsilon=\nu^{3} / \eta^{4}$, and the Taylor microscale is defined by $\epsilon=15 \nu u^{\prime 2} / \lambda^{2}$. The energy dissipation, reduced by integral scales, is $\epsilon \Lambda / u^{\prime 3} \sim 6.5 / \sqrt{R e_{\lambda}}$, for $R e_{\lambda}<100$. Their Figure $2 \mathrm{~b}$ indicates non-isotropic behavior for the small scales which may be caused by the deterministic forcing scheme (the negative viscosity imposes a $\nabla^{2}$ pattern). Large-scales creating deterministic small-scale structure has been examined by Yeung \& Brasseur [20].

From distributions of vortex size and circulation, they find intense vorticity with an apparent Gaussian radial distribution, the 1/e radius is approximately $4 \eta$, and the estimated circulation is $\Gamma \approx 22 \sqrt{u^{\prime} \lambda \nu}$. They suggest that a square-root behavior develops from shear layers that have a velocity difference of $u^{\prime}$ and a layer thickness that has been squeezed down to the Kolmogorov length. This gives $u^{\prime} \sim \Gamma / \eta$ and so $u^{\prime} \eta / \nu \sim \Gamma / \nu$, and with the relation $\lambda / \eta \sim 2 \sqrt{u^{\prime} \lambda / \nu}$, they obtain

$$
\frac{u^{\prime} \eta}{\nu} \sim \frac{\Gamma}{\nu} \sim \sqrt{\frac{u^{\prime} \lambda}{\nu}}
$$

and thus, pieces of these thin shear layers that roll up into vortex tubes will have the square-root circulation behavior. They comment that these tubes appear on the edges of regions which are nearly uniform in their velocity values - this feature would imply that collisions between fluid volumes creates shear layers which sometimes roll up into vortex tubes. This mechanism may require an oblique impact angle between the two fluid volumes according to Ruetsch [21]. The collision process may create an extensional strain rate in the direction of the vorticity, and it is this axial strain rate which can maintain the Burgers' vortex as a steady, viscous solution. As a footnote in a 1939 meeting paper [22], Burgers presented this solution in response to Taylor's 1938 paper which stated that vortex stretching might be important in the dissipation of mechanical energy. Replacing the time variable in Oseen's vortex with an axial strain, Burgers shows the balance between strain rate $s$ and kinematic viscosity $\nu$ creates a Gaussian radial distribution of vorticity which creates a swirling velocity around the vortex axis of

$$
u_{\theta}=\frac{\Gamma}{2 \pi r}\left[1-\exp \left(-s r^{2} / 4 \nu\right)\right]
$$

where $r$ is the radial distance. A constant axial strain rate of $u^{\prime} / \lambda$ would be consistent with the 1 /e radius of $4 \eta$. When the axial strain ceases, then the vortical core expands, but 
expands slowly as $\sqrt{4 \nu t}$, and while it is expanding, any radial location outside the decaying core still responds to the circulation $\Gamma$ contained within that radial location. Therefore, while the vortex is decaying, the swirling flow outside the decay region does not yet realize that the axial strain race has disappeared.

The maximum swirling velocity $U_{\theta}$ produced by the Burgers' vortex is close to the $1 /$ e radial location, and with $u^{\prime} \eta / \nu=\sqrt{u^{\prime} \lambda / 4 \nu}$, we obtain

$$
\frac{U_{\theta}}{u^{\prime}}=\frac{0.63 \Gamma / \nu}{8 \pi \eta u^{\prime} / \nu}=\frac{0.63\left[2(\Gamma / \nu) / \sqrt{u^{\prime} \lambda / \nu}\right]}{8 \pi} \approx 1
$$

when $\Gamma=22 \sqrt{u^{\prime} \lambda \nu}$ is used. Thus, the maximum swirling velocity is the rms velocity! This observation may explain why much of the experimental turbulent flame speed data has $S_{T} / S_{L} \sim u^{\prime} / S_{L}$, because this swirling motion around the vortex tube axis creates flame area, see Figures 2 and 3. This intense vortex tube structure and its effect on flame shape and propagation is the essential concept of this review.

Flow around a vortex may also describe a behavior found in direct simulations of particles within a turbulent flow. Yeung \& Pope [23] computed trajectories of Lagrangian particles and they found the time correlation of an acceleration component goes through zero in 2.2 Kolmogorov time units, independent of Reynolds number. If the particle is going around the vortex axis, at the maximum swirling radius, then the acceleration would correspond to the centrifugal force and this acceleration in Cartesian coordinates would appear as sinusoidal in time - the period from maximum to zero would be one-quarter of a rotation around the vortex axis. The time for one rotation is $t_{u}=2 \pi R / U_{\theta}$, where $R$ is the location of maximum swirling velocity; $\approx 4 \eta$. Scaling one-fourth of this time with the Kolmogorov time $\tau_{\eta}$ gives $t_{u} / 4 \tau_{\eta}=\pi R e_{r \theta} / 2 U_{\theta}$, where we assume that $\tau_{\eta} e_{r \theta} \sim 1$. The shear $e_{r \theta}$ and the angular velocity $U_{\theta} / R$ have the same dependence upon $\Gamma / R^{2}$ and so will have the same Reynolds number dependence. Therefore, this acceleration time period has no Reynolds number dependence.

Another particle behavior that might be related to vortex structure is the condensation of Stokes particles within computed turbulence [24]. Capture of a particle might result from the balance of inward strain rate normal to vortex axis and the outward centrifugal force. A certain range of Stokes number would allow the particle to ride around the vortex at the maximum swirling location. Smaller values would spiral inward and larger values would be affected by neighboring structures. Fully interacting simulations, but with decaying turbulence have been performed in [25] and within a two-dimensional periodic vortex field 
the dynamics of buoyant and heavy particles has been simulated [26].

\section{Summary of Structure}

The numerical simulations of turbulence allow examination of microscopic flow patterns which affect turbulent combustion. These simulations are large enough to show agreement with experimental results and so models of the vortical structure should be developed in order that their effect upon turbulent combustion can be modeled. Turbulent combustion models based on the energy-cascade concept of Kolmogorov have been reviewed by Bray \& Cant [27], in the following, we give new concepts about propagation through this vortical structure and the resulting turbulent flame speed models that can be created.

\section{Flamelet Models}

To investigate flame stability, Markstein wrote a flame evolution equation in 1964, but without a nonlinear tool he was restricted to a stability analysis [28]. Sethian developed a technique [29] to follow a front as one level surface in a continuous field and thereby topology changes are easily treated. Passive front propagation through a flow structure can be done if volume expansion is ignored, but these constant-density flames appear so artifical that one hesitates to use them, however, we find they reveal concepts of turbulent propagation. Therefore, the flamelet assumption has been exploited in order to decouple the complexity of chemistry from that of turbulence. Markstein's notation was $f$ for a flame with volume expansion; while a passive formulation [30] has acquired the letter $G$; we distinguish between these flamelet models by using $f$ when volume expansion is considered and $G$ when it is not. The special initial condition formulated by Kerstein is $G=x$ for the evolution equation

$$
\frac{\partial G}{\partial t}+\mathbf{u} \cdot \nabla G=S_{L}|\nabla G|
$$

where the right side describes Huygens' propagation. This nonlinear term makes the initial condition very special: it provides a connection between flame area and the gradient of $G$. The scalar gradient corresponds to the flame surface density, that is, flame area per unit volume. This formulation allows a numerical simulation which follows Damköhler's idea: creation of flame area by turbulent motion causes the larger consumption rate. Now, with the passage of fifty years, the computer tool can easily determine passive flame area within a turbulence simulation and the motion can be forced so that a statistically steady propagation is obtained. 
The G-Equation and the Z-Equation

Flamelets within nonpremixed combustion have a long history and now with the scalar $G$-equation, Peters [31] has unified the procedure to obtain a one-dimensional temperature equation for premixed and nonpremixed reaction, the latter using the mixture fraction $Z$ and the former using the $G$-equation. The temperature equation for a premixed flamelet is obtained by a transformation from physical space to $G$ space. Using a coordinate normal to the flame surface, $x_{n}=G /|\nabla G|$ and the propagation equation, the one-dimensional temperature equation is

$$
\rho \frac{\partial T}{\partial t}+\rho S_{L} \frac{\partial T}{\partial x_{n}}=\frac{\partial}{\partial x_{n}}\left(\rho D \frac{\partial T}{\partial x_{n}}\right)+w
$$

where the burning velocity $S_{L}$ replaces the convection velocity and $w$ is the reaction term. For premixed combustion, this results in a flame zone that should be thin in physical space, while for nonpremixed reaction, the chemistry should occur within a narrow, range of mixture fraction.

Neither flamelet equation has a chemical source term. Since $Z$ is a conserved scalar with respect to chemical reaction, it is clear why there is no source term for $Z$. But this is less clear for the $G$-equation where the gradient of $G$ times the burning velocity appears. This scalar has been created in order to track a front, the scalar gradient defines the flame normal as $\mathbf{n}=-\nabla G /|\nabla G|$ and is used in the kinematic balance describing Huygens' propagation. This scalar gradient has a smooth variation through the thin reaction zone and so, in this sense, the right side of the G-equation is not a chemical source term but it is a kinematic source term for Huygens' propagation. Note that this propagation is not a diffusive one and therefore there is no diffusive term in the G-equation.

We now describe $G$-equation simulations with computed three-dimensional NavierStokes turbulence, the turbulent kinetic energy has been maintained at a constant value so that steady propagation can be observed within a periodic domain.

\section{Geometry of the G-Surface}

The curvature of computed $G$ surfaces has been taken in two-dimensional slices that mimic laser produced flame images. Comparison throughout a turbulent flame zone [32] shows that one length scale normalizes experimental results into agreement with computed curvature distributions. The mean positive curvature does not change with location, but the mean negative curvature increases with distance from the leading edge of the zone. 
A full surface definition is given by the normal vector $\mathbf{n}$ and two eigenvectors of curvature $\mathbf{h}_{1}, \mathbf{h}_{2}$, where $\left|h_{1}\right| \leq\left|h_{2}\right|$. The ratio of these two eigenvalues $h_{1} / h_{2}$, the shape factor introduced by Pope [27], indicates the most likely shape is that of a cylinder. This cylindrical feature is caused by the swirling velocity around the intense vorticity, Figure $\mathbf{3}$ shows how this swirling motion distorts the flame and creates an alignment between $\mathbf{h}_{\mathbf{1}}$ and the vorticity vector [33]. Previous results have shown that vorticity aligns with the intermediate strain direction $\beta$ (where the eigenvalues are $\alpha \geq \beta \geq \gamma$ ) and Figure 4 shows that the alignment probability of the flame cylinder axis is very similar to the alignment of intermediate strain with vorticity. The extensional strain rate in the plane of the flame is usually smaller than the rms estimate of $\sqrt{7.5 u^{\prime 2} / \lambda^{2}}$, the former is closer to $u^{\prime} / 2.5 \lambda$.

\section{G-Fluctuations and the Mean Progress Variable}

The initial condition $G=x$ allows fluctuations of $G$ to describe the flame spatial displacement and this information can be related to the mean progress variable and so, contact with flamelet models based on a progress variable can be made [27]. In the $G$ simulation, the initial mean gradient is maintained with a jump periodic boundary condition in the $x$ direction, the mean propagation direction. The average of $G$ over a constant $x$ plane is $\left\langle G\left(x_{p}\right)>\right.$ and scalar fluctuations on that plane, $G^{\prime}=G\left(x_{p}, y, z\right)-\left\langle G\left(x_{p}\right)\right\rangle$, correspond to flame displacement from its mean location $G^{\prime}=-x_{d}$, where $x_{d}$ is the displacement of the surface. From simulations [32], the probability distribution of scalar fluctuations determine the mean progress variable as

$$
\bar{c}(x)=\int_{G^{\prime}=-x}^{\infty} P\left(G^{\prime}\right) d G^{\prime}
$$

where the total integral of the probability density $P\left(G^{\prime}\right)$ is unity. The value of $\bar{c}(x)$ can also be computed by measuring the displacement of selected $G$ surfaces; the direct result agrees with the $G^{\prime}$ result. 'i'he mean progress variable is also given by a travelling wave solution to a nonlinear reaction diffusion mechanism, the KPP equation

$$
\frac{\partial \bar{c}}{\partial t}=w \bar{c}(1-\bar{c})+D \frac{\partial^{2} \bar{c}}{\partial x^{2}}
$$

where $w$ is the reaction rate and $D$ the turbulent diffusivity. A first approximation [34] for the spatial gradient of the mean progress variable is

$$
\frac{d \bar{c}(x)}{d x}=a \bar{c}(x)[1-\bar{c}(x)]
$$


and in the full numerical solution the variation in the $a$ coefficient is only noticeable near $\bar{c} \sim 0$. Therefore, with a constant coefficient, the spatial distribution is $\bar{c}(x)=$ $[1+\exp (-a x)]^{-1}$ and by differentiation of $\mathrm{Eq}(6)$, the approximate distribution for scalar fluctuations is

$$
P\left(G^{\prime}\right)=\frac{a \exp \left(a G^{\prime}\right)}{\left[1+\exp \left(a G^{\prime}\right)\right]^{2}}
$$

and this approximation agrees with the simulation results. These $P\left(G^{\prime}\right)$ distributions have a larger probability near the origin than the Gaussian distribution. These $G$ simulations, which are based on Huygens' propagation into the unburnt gas, give ensemble results which are well described by the reaction-diffusion balance in the KPP model. Yet the microscopic flame dynamics are not diffusive! Therefore, even though the ensemble behavior is given by a diffusive model, diffusion-based models of microscopic dynamics may give the wrong combustion dynamics. Hence, in a Large Eddy Simulation, how large must the space and time domain be for a diffusive sub-grid model versus a non-diffusive one?

\section{Partially Premixed Flamelets}

Early injection of fuel into the Diesel engine may create regions of partially premixed fuel and air and after ignition there will be flame propagation through the regions which are connected and within the flammability limits. The remaining charge may be considered as a nonpremixed reaction. The burning velocity exhibits a large variation within the flammability limits and this variation may affect the premixed flame shape. Near stoichiometric conditions the flame normal should be normal to the mixture fraction gradient, but near lean and rich pockets the gradient of $G$ aligns with the gradient of $Z$. Propagation along a stoichiometric contour may be sensitive to heat loss when the flame dimension normal to the contour approaches the flame thickness, a gap quenching mechanism might occur. Ignoring some of these complications at this time, Peters. [31] has formulated a passive partially premixed flame propagation based on the $G$-equation and the $Z$-equation. From the known behavior of $S_{L}(Z)$ the Huygens' propagation is simulated in a $Z$ field which has a constant variance in addition to a constant turbulent kinetic energy. Pockets of $Z$ which are too rich or are too lean in comparison to $Z_{s t}$ retard the local propagation of a $G$ surface. Some dynamics of these pockets will depend on volume expansion effects which are not included in this passive formulation. The burn out of a rich pocket should be important in soot production.

\section{G-Equation Applications}


Collins [35] has used two-dimensional spectral simulations to evaluate the analysis given by Peters [36] for the spatial correlation of scalar fluctuations. By choice, the forcing style suppresses coherent flow structures and the location of the forcing wavenumber creates an energy spectrum with either a $-5 / 3$ or a -3 power-law. In the $x, y$ domain, the mean scalar gradient is enforced by using $G=x+g$, where $g$ is the scalar fluctuation and it is periodic in all directions. The propagation equation is

$$
\frac{\partial g}{\partial t}+w^{\prime}+\mathbf{u} \cdot \nabla g=S_{L} \sqrt{\left(\frac{\partial g}{\partial x}+1\right)^{2}+\left(\frac{\partial g}{\partial y}\right)^{2}}
$$

where $w^{\prime}$ is the velocity fluctuation in the mean propagation direction $(=\mathbf{u} \cdot \mathbf{i})$. The mean scalar gradient makes anisotropic scalar fluctuations via the $w^{\prime} w^{\prime}$ term in the equation for $\left\langle w^{\prime} y\right\rangle$. This makes $\left\langle w^{\prime} g\right\rangle$ negative in value and it is the principal source term for the scalar spatial correlation $\langle g(\mathbf{x}) g(\mathbf{x}+\mathbf{r})\rangle$. Peters [36], wishing to apply established analytical methods for isotropic scalar fields, did not include this anisotropic source term in his two-point correlation analysis. He considers a field in which flamelets travel from all directions into a certain spatial domain, and then by assumption, the mean gradients cancel. Peters used the propagation equation given in Eq. (4) and split the velocity and $G$ into mean and fluctuation components. To explore this isotropic assumption, consider an ensemble of flamelets, with propagation direction for the $j^{\text {th }}$ flamelet given by unit vector $\mathbf{e}_{\mathbf{j}}$, and use the propagation equation

$$
\frac{\partial g}{\partial t}+\mathbf{u} \cdot \mathbf{e}_{\mathbf{j}}+\mathbf{u} \cdot \nabla g=S_{L} \sqrt{1+2 \mathbf{e}_{\mathbf{j}} \cdot \nabla g+\nabla g \cdot \nabla g}
$$

with the initial condition that $g$ is zero; $G(\mathbf{x}, t)=\mathbf{e}_{\mathbf{j}} \cdot \mathbf{x}+g(\mathbf{x}, t)$. It would be interesting to compare scalar statistics in the unidirectional field with those $c f$ an isotropic field.

The current simulations by Collins yield a scalar spectrum with a power-law behavior and the power depends upon the shape of the energy spectrum. The scalar spectrum obtained by Peters has additional exponential factors which are not seen in these simulations, perhaps due to the limited range of the simulations. Wirth \& Peters [37], by assuming that $\partial g / \partial x$ can be neglected in comparison to unity in the Huygens term of Eq (10), have obtained the scalar spectrum from two-dimensional flame images within an engine. This assumption is not always valid, but is useful since it allows flame images to be converted into scalar correlations and hence the scalar spectrum.

Zhu and Ronney [38] have performed what they call a Huygens-DNS simulation (specified flame velocity within a computed flow) of a Taylor-Couette flow with a single scale 
of counter-rotating eddies. This flow with liquid flames can produce propagation with $u^{\prime}$ a thousand times $S_{L}$ and the resulting observed behavior is $S_{T} \ln \left(S_{T} / S_{L}\right) \sim u^{\prime}$. This relationship indicates a flame perimeter that grows in proportion to its length over the time period in which the advancing flame engulfs the vortex; that is $d L / L \sim d t / \tau$, where $\tau \sim L / u^{\prime}$ is the eddy rotation time and the engulfment time period is $L / S_{T}$. The computed and experimental flow-flame pattern exhibits a diminishing row of unburnt pockets behind the leading front, when $u^{\prime} \gg S_{L}$, but the flame advancement appears to depend upon how the flame reaches the next eddy; thus, variations in the shape of the computed-eddy does yield large variations in $S_{T}$.

\section{Turbulent Flame Speed}

Is there a generic "Turbulent Flame Speed" or is it the net result of many interacting effects? Consider the possible effects in the reaction-sheet regime: the laminar burning velocity $S_{L}$, the volume expansion $T_{b} / T_{u}$, the turbulence structure $u^{\prime}, \lambda$, the change in $S_{L}$ due to strain rate and curvature, the Lewis number, the flame shape and size and the flow

confinement. Variations in the unburnt gas flow structure, which have usually not been measured, may explain part of the historical confusion regarding turbulent flame speed data. Another part is knowing the actual mass flux which is entering the flame zone. For example, the double kernel method has been used in a fan-stirred chamber with the assumption [39] that as the two flames meet, they will approach each other at twice the $S_{T}$ value. However, if the flow induced by volume expansion is considered, then as Shepherd illustrates [40], the mass flux away from the line joining the two flames is not entering the flames, and so this method yields an overestimate of $S_{T}$. Variations in flame shape and size as the two flames meet could produce large variations in the ignored mass flux and this may partly explain the large scatter in the $S_{T}$ values given by this technique. The concept of a turbulent flame speed has generated many model approaches, and many functional forms - most have been based on the Kolmogorov cascade concept and not on particular flow patterns creating flame area. Within the engine community, the current practice is a two-zone model of flame propagation; the burnt zone is separated from the unburnt zone by a spherical surface and a simple cut-off is used where the sphere extends beyond the piston-cylinder walls. Thus, volume expansion is not allowed to interact with the walls and deform the flame surface (the tulip flame deformation has been numerically examined by Gonzalez et al. [41]). These methods require a turbulent flame speed model and we now describe some of the current models. 


\section{Engine Combustion Models}

The February SAE meeting papers and the work presented at the KIVA Users meeting reveal the current models used in engine combustion analysis. Commerical codes are available, and with $10^{4}$ to $10^{5}$ elements, very realistic engine flow geometry can be computed. Computation times range from hours to tens of hours. The turbulence model is usually $k-\epsilon$ and the Magnussen model of $\epsilon / k$ for the reaction rate is a popular choice. The next level of flame description is the Coherent Flame Model which uses a balance equation [42] with assumed flame area growth and destruction term:3. Another approach is the Fractal Flame Model which uses the experimental fractal dimension of flame contours to produce the total flame area [43]. Bielert et al. [44] have constructed a two-dimensional flame model in which the leading edge of the flame front is tracked and consumption of eddies behind this front, over a finite time period, gives a turbulent flame zone with finite thickness. Concentrating on only the early flame growth, and using measurements of flame radii from 3 to $11 \mathrm{~mm}$, Boulouchos et al. [45] have constructed a spectral model which allows the range of flow scales that create flame area to grow with the flame size. They also use a 2-step model: laminar growth with a step change to turbulent propagation. Both these models can describe the rather sparse data set of flame radii. They have also considered the Gibson scale idea - that scale being the minimum scale that wrinkles the flame - but they find it gives unrealistic behavior over the conditions of the data set.

Within the engine community there has been a debate on the existence of a steady turbulent flame speed. To answer this question, Checkel has constructed a five inch cube [46], within which turbulence is created by pushing a perforated plate through the chamber before ignition. The flame radius can then be observed as a function of time. The results of Ting et al. [47] reveals continual flame speed growth for flame radii up to $55 \mathrm{~mm}$, and so for dimensions typical of a car engine, the turbulent burning rate does not seem to have a constant value. A model of this engine-type propagation has been developed [48] and even though the current model may not be correct in all aspects, the concepts do illustrate how flamelet simulations, combined with turbulence simulations and experimental information, can lead to a propagation mechanism that is based on realistic flow patterns rather than assumed flow concepts.

\section{Flame Speed and Flame Size}

The experimental results yield a turbulent flame speed which increases with the size of the flame ball, whereas a spherical laminar flame ball has a constant speed of $S_{L}+\tau S_{L}$, 
the first term is the burning velocity advancement into the gas ahead of the flame and the second term is the velocity created by volume production, the heat release parameter $\tau$ is the temperature ratio minus unity. The turbulent flame speed growth with size is a consequence of volume expansion distributed throughout the flame ball. The velocity field created by volume expansion confined to a spherical surface, with radius $R_{s}$, is

$$
V_{s}=\tau S_{L} \frac{R_{s}^{2}}{R^{2}}
$$

for $R \geq R_{s}$; there is no velocity effect within the sphere and the surface velocity uoes not depend upon size. Now, if the flame is wrinkled then the volume expansion effect at the largest flame radii will be different than at smaller radii. Similar to a continuous flow burner, where the turbulent flame density is given by $d \bar{c} / d x$, we assume a spherical shell which contains a constant flame density. The volume production is reiated to this flame surface density and we assume it is proportional to

$$
\frac{d \bar{c}}{d x} \sim \frac{1}{2 \pi \lambda} \sqrt{\frac{u^{\prime}}{S_{L}}}
$$

based on passive $G$-equation simulations. When $u^{\prime}>S_{L}$, the heat release should limit the flame area, but this effect is neglected. The inner radius $R_{i}$ and outer radius $R_{o}$ define the flame zone and this distributed volume production will create a flow velocity of

$$
V_{R}=\tau S_{L} \sqrt{\frac{u^{\prime}}{S_{L}}} \int_{R_{i}}^{R_{\mathrm{o}}} \frac{R_{s}^{2}}{R^{2}} \frac{d R_{s}}{2 \pi \lambda}
$$

for $R \geq R_{o}$. Integration gives

$$
V_{o}=\frac{\tau S_{L} R_{o}}{6 \pi \lambda} \sqrt{\frac{u^{\prime}}{S_{L}}} \quad\left[1-\left(R_{i} / R_{o}\right)^{3}\right]
$$

for the flow velocity at the largest flame radius $R_{o}$. When the inner radius is a small fraction of the outer radius - a thick flame zone, then the velocity of the outer flame ball will depend upon the flame size. However, if the flame zone is thin $-R_{i} \sim R_{o}$, then the bracket ter:m will vanish and the velocity will be independent of flame size as in the laminar case. The turbulent flame speed estimate is

$$
\frac{S_{T}}{S_{L}}-1 \sim C_{R} \frac{R}{6 \pi \lambda} \sqrt{\frac{u^{\prime}}{S_{L}}}
$$

where $C_{R}$ is the bracket term value. Now, we replace the Tylor scale with the integral scale $\Lambda$ and find a linear dependence upon the ratio $u^{\prime} / S_{L}$. Using $\Lambda / \lambda=\sqrt{u^{\prime} \Lambda / 15 \nu}$; 
and replacing the kinematic viscosity with the thermal diffusivity, $\nu=0.7 \alpha$; and using an estimated flame thickness, $\delta_{f}=7.4 \alpha / S_{L} ;$ we obtain

$$
\frac{S_{T}}{S_{L}}-1 \sim \frac{0.045 C_{R} R}{\sqrt{\Lambda \delta_{f}}} \frac{u^{\prime}}{S_{L}}
$$

and so, the linearity in $u^{\prime} / S_{L}$ is the product of two square-root terms. The data indicates that $C_{R}$ is near unity which implies that $R_{i}$ is about half the flame radius if the $2 \pi \lambda$ factor is correct. A flame zone that grows, $L \sim R_{o} / 2$ also implies a constant burnout time, as used in [44].

In a numerical study of flame ball development, Kwon et al. [49] assumed that the velocity field created by volume expansion confined to a single sphcical surface could be combined with velocity fluctuations in order to simulate the flame dynamics. This single sphere with a thin flame zone assumption restricted their results to a turbulent flame speed that does not grow with flame size except through the creation of extra flame area by the fluctuation velocities.

How many phases does the flame ball growth have? After spark ignition, the early flame surface may encounter flow structures which are large in comparison to the burnt volume at that instant and so, the flame-flow interaction in that case may not resemble the spherical velocity distribution used above. After the flame ball has developed to a size where there are many flow structures interacting with the flame at once, then the uniformly distributed volume source solution may be reasonable. Changes from the spherical shape are observed indicating that the spherical shape may not be a stable one. Indeed, an ellipsoid will create a larger flow velocity in the major axis direction, but while making the flame motion faster, this expanison effect will increase the unburnt gas divergence and this may restore the spherical shape. As mentioned above, the heat release parameter could also determine an upper limit for the flame surface density.

\section{Steady, Planar Flame Speed}

Given a spherically expanding flame, can we extract a value for steady propagation in other configurations? Normalizing the flame radius with $\sqrt{\lambda \Lambda}$, the geometric mean of the two length scales which can distort the flame surface, we have from Eq. (16)

$$
\frac{S_{T}}{S_{L}} \sim \frac{R}{\sqrt{\lambda \Lambda}} \sqrt{\frac{u^{\prime}}{S_{L}} \frac{\Lambda}{\lambda}}
$$

and the last term, by itself, collapses experimental data of steady, planar turbulent flame speeds [50]. Changing the length scale ratio into $R e_{\lambda}$, this same relation fits the data of 
Liu \& Lenze [51]

$$
\frac{S_{T}}{S_{L}}-1=1.75 \sqrt{\frac{u^{\prime}}{S_{L}} \frac{R e_{\lambda}}{15}}
$$

and, by making small changes in some of their $\lambda$ estimates, the above relation describes all their data points. A different estimate, but also based on passive $G$-equation simulations is given by Wirth \& Peters [37] as

$$
S_{T}=\left(S_{L}+0.8 \sqrt{S_{L} u^{\prime}}+1.5 u^{\prime}\right)\left(1-1.4 \frac{L_{M}}{\Lambda} \frac{u^{\prime}}{S_{I}}\right)
$$

where the square-root term is the G-equation result and the term with $u^{\prime}$ is the assumed large intensity limit, but modified by the last factor which includes the flame thickness compared to the flow length scale.

$S_{T}$ versus $u^{\prime}$ - the bending

We now discuss the "bending" feature of $S_{T}$ vs $u^{\prime}$. The linearity of $S_{T}^{\prime}$ with respect to $u^{\prime}$ may depend on how the length scales change as $u^{\prime}$ is increased in an experimental configuration. The passive $G$-equation simulations imply that variation of $S_{T}$ with $u^{\prime}$ depends upon $S_{L}$ and $R e_{\lambda}$, where the Reynolds number dependence is related to flame propagation through a non-space filling vortical structure [52]. Thus, the bending effect could be a structural effect of the turbulence. This vortical structure concept differs from that of Bradley [39], who considers bending to be a strain rate reduction of $S_{L}$ and so, flame stretch is the key parameter which causes bending. The Karlovitz stretch factor is

$$
K=0.157 \frac{\left(u^{\prime} / S_{L}\right)^{2}}{\sqrt{R e_{\Lambda}}}
$$

but using this stretch relation to define $R e_{\lambda}$ in Eq. (19), we find agreement [48] with the summary plot of $S_{T}$ given in Figure 4 of [39] for $u^{\prime}<6 S_{L}$. Thus, flame stretch may not be the only cause of bending. Models which incorporate the statistical behavior of flame stretch found in simulation results are given by Kostiuk \& Bray [53].

Another possible contribution to bending is the reduction in flame folding when volume expansion is included, that is, the flame surface will interact with itself through the BiotSavart interaction and this interaction will inhibit the folding seen in a passive flame simulation. Additionally, the flame shape may induce a mass flux away from the flame, and thereby reduce the burning rate.

To resolve the relative importance of vortical structure versus strain rate versus volume expansion, we need simulations with heat release and over a range of Reynolds numer, say 
$30<R e_{\lambda}<150$ with values of $u^{\prime}$ up to ten times the burning velocity. The topics to be explored are: 1) As $R e_{\lambda}$ is increased, does the vortical structure have gaps that cause bending, or are there many little tubes that fill in the gaps and yield a more linear behavior in $S_{T}$ vs $u^{\prime}$ ? 2) Given this vortical structure, for various mixture fractions and Lewis numbers, how large is the effect of flame stretch upon the total flame area? 3) How does total flame area change with increasing heat release?

\section{Vortex-Flame Interaction}

Creating a known vortex structure and letting it interact with a flame has recently been performed by several groups. Santavicca et al. [54] have used a V-shaped flame interacting with a Kárrián vortex street. Ground state $O H$ is imaged in a plane of laser light. Samaniego [55] has puffed a vortex pair from a slot into a V-shaped flame and observed a quenching, re-ignition process. Roberts et al. [56] have created vortex rings which meet a propagating flame and determined the vortex strength/size combinations which wrinkle the flame and those which locally quench the flame. Their definition of quenching is any local flame section that exhibits a peak $O H$ intensity which is less than 0.01 of the undisturbed flame intensity. Their configuration is a vertical tube with premixed methane-air in which a nearly flat flame propagates upwards while the vortex ring moves down, the resulting interaction may quench the flame, create pockets of unburnt, or not even wrinkle the flame. The observed minimum vortex size that wrinkles the flame is $U_{\theta} / S_{L}=2.5 \delta / d_{c}$, where the flame thickness $\delta$ is estimated as being $7.4 \alpha / S_{L}$, with $\alpha$ the thermal diffusivity, and the estimated core size $d_{c}$ is $0.2 D$, where $D$ is the orifice diameter that creates the ring. To quench a thin flame, the effect of flame stretch upon quenching is thought to be a function of the Karlovitz relation, which for this vortex ring case is $\left(U_{\theta} / a_{c}^{\prime}\right)\left(\delta / S_{L}\right)$. However, as the vortex size is changed, their single vortex-ring quenching data does not exhibit a constant value of this Karlovitz parameter until the core size becomes large, $d_{c}>10 \delta$, and then the value is 2.2 . They conclude that small vortices are not as effective in quenching the flame as the Karlovitz concept would predict.

However, a Péclet number is constant along their quenching boundary. We see that the vortex ring parameters that cause quenching are $U_{\theta} / S_{L} \sim 9.2 \delta / d_{c}$ and this expression is also a Péclet number by replacing the flame thickness with $7.4 \alpha / S_{L}$. Thus, the quenching Péclet number is: $U_{\theta} d_{c} / \alpha=68$. The ratio of $\left(U_{\theta} / d_{c}\right)$ to $\left(d_{c}^{2} / \alpha\right)$ could reflect a flow time scale relative to a heat transfer time scale. In summary, in their single vortex ring flame interaction, a Péclet number below 20 causes no flame distortion and a Péclet number 
above 70 creates a local quenching. Noteworthy is that a two-dimensional, single reaction step flame simulation has the same quenching trends as seen in the experiments, and therefore, more study of this flow should provide the quenching concepts for this vortexflame interaction.

A direct numerical simulation of two-dimensional, decaying turbulence interacting with a $\mathrm{H}_{2} / \mathrm{O}_{2} / \mathrm{N}_{2}$ system has been performed by Baum $\epsilon_{i}$ al. [57]. They have selected the nine-species, 19-reaction scheme devised by Miller, Mitchell, Smooke \& Kee and so, this should be considered a "full" kinetics flow simulation by comparison to previous efforts. While very costly in computer resources, this type of work provides a standard against which reduced-kinetic models may be judged. For example the peak $H$-atom is near the flame, i.e. the peak heat release, but there is a long diffuse region in the products. The peak $O H$ is well behind the flame and does not remain as a connected contour, and so images of this species would not be a valid experimental indicator of the heat-release connectivity.

These simulations with volume expansion require inflow/outflow bounday conditions, and as discussed by Poinsot [58], the compressible formulation with its explicit tracking of acoustic wave motion allows an easier boundary treatment. The acoustic time-step restriction does make this formulation more costly if a near-zero Mach number system is considered, but acoustic effects are needed if combustion stability questions are to be included. Currently, simulations in two-dimensions which include detailed chemistry and heat release are at the limit of computer capability, and therefore are done for that reason [58]. However, as discussed by Collins [35], details of the forcing scheme may encourage the formation of vortical flow structures and it would be good to exploit the two-dimensional system so that it mimics some features of the three-dimensional vortex structure. For example, as a function of Reynolds number, the vortex circulation and core diameter and the spacing between the vortices could be made to match the three-dimensional structure. 


\section{The Turbulent Future}

When will the turbulence problem be solved? Since the flow is a nonlinear problem, then progress before the computer, the tool for developing nonlinear solutions, was limited. Additionally, before the laser-slice technique, the experimental information was also obscured by line of sight information and pointwise, random looking signals. The growth in the computer tool has occurred during my career: thirty years ago I used an IBM 1620 with card input and card output, twenty years ago I used a thousand CDC 6600 hours to generate molecular dynamics simulations that reproduced the shear viscosity of liquid argon, ten years ago other people were using fifty hours on a CRAY-1 to simulate turbulence with a million grid points, today the CM-2 produces flow fields with 64 Giga-bytes of information, and ten years from now turbulence will be solved! Maybe sooner, maybe later - but the explosive growth in the nonlinear tool, combined with its widespread use and combined with experimental flow images, means that someone will create, a model with the right perspective and thereby capture the essence of turbulent combustion.

For premixed flame propagation, where do we stand? Will we understand the dynamics of the reaction-sheet regime? For passive propagation, I think the understanding has started with the use of the $G$-equation, the constant energy turbulence simulations allow a statistically steady flame motion to develop, and the probing of that flow allows models to be built and hence understanding gained. Currently, we find that a two-dimensional flame-vortex interaction is a good configuration for exploring the effects of turbulence upon detailed flame structure. Going beyond these passive simulations requires the inclusion of heat release, and this calls for an adaptive grid in order to resolve the desired thin flame zone with large density change. The questions to be answered are: Does the baroclinic production of vorticity change the flame surface geometry in a significant way from the passive flame? Does angular momentum within the vortex tube respond to the density change across the flame in ways that change propagation? Does the volume expansion affect the unburnt vortical structure? Does the pollutant formation depend upon flame shape? Does the burnt gas heat transfer and mixing depend upon the hot vorticity structure? 


\section{Acknowledgements}

This work supported by the United States Department of Energy through the Office of Basic Energy Sciences, Division of Chemical Sciences. Their support of fundamental work without imposing specific shortrange goals has allowed a very open approach, and by looking back, one can see many interconnections that were not visible when thinking ahead.

I met Norbert Peters at the 1979 Shear Flow conference, and while I was 48 hours ahead at birth, he clearly has led me into the combustion world. During a 1980 CRF research visit, Bob Bilger suggested that the premixed problem was in great need in comparison to nonpremixed. Interactions with Forman Williams started in 1980 and have always been enjoyable and encouraging, as have the many trips to Cal Tech since the 1983 invitation by Paul Dimotakis. Alan Kerstein joined the group in 1981 and the many wild-idea, bad-pun sessions produced sol.e of the work given above. By sharing their computer results, Parviz Moin \& Mike Rogers got us into the DNS world. By creating the Center for Turbulence Research, Moin \& Reynolds will continue to aid the whole combustion community.

\section{References}

1. Williams, F. A., Twenty-Fourth Symposium (International) on Combustion, The Combustion Institute 1 (1992).

2. Liñán, A. \& Williams, F. A., Fundamental Aspects of Combustion, Oxford Univ. Press (1993).

3. Chen, J. H., Turbulent Shear Flows 8, p 297 (Springer-Verlag, 1992); Chen, J. H. et al. Proc. Summer Program 1990, Center for Turbulence Research, NASA-Ames/Stanford University, compare Figure 10 with 14.

4. Roshko, A., Instability and Turbulence in Shear Flows. in Theoretical and Applied Mechanics 1992, Bodner, S. R. et al. editors (Elsevier Science Publishers B. V., 1993).

5. Boratav, O. N., Pelz, R. B. \& Zabusky, N. J., Phys. Fluids A 4, 581 (1992).

6. Karasso, P. S. \& Mungal, M. G., LIF Measurements of Mixing in Turbulent Shear Layers. Sixth Inter. Symp. an Applications of Laser Techniques to Fluid Mech., Lisbon, Portugal, July 1992; also Karasso, P. S., thesis "Experiments on Scalar Mixing and Reaction in Plane and Curved Turbulent Shear Layers," Stanford University, 1994.

7. Lopez, J. M. \& Bulbeck, C. J., Phys. Fluids A 5, 1694 (1993); erratum 3013.

8. Liepmann, D. \& Gharib, M., J. Fluid Mech. 245, 643 (1992).

9. Abid, M. \& Brachet, M. E., Phys. Fluids A 5, 2582 (1993).

10. Hertzberg, J. R. \& Ho, C. M., AAIA Jour. 30, 2420 (1992).

11. Chao, Y.-C. \& Jeng, M.-S., Twenty-Fourth Symposium (International) on Combustion, The Combustion Institute 333 (1992).

12. Lasheras, J. C., Liñán, A., Lecuona, A. \& Rodriguez, P., Twenty-Fourth Symposium (International) on Combustion, The Combustion Institute 325 (1992).

13. Edwards, C. F. et al., Fuel 72, 1151 (1993).

14. She, Z.-S., Jackson, E. \& Orszag, S. A., Nature 344, 226 (1990).

15. Trefethen, L. N., Trefethen, A. E., Reddy, S. C. \& Driscoll, T. A., Science 261, 578 (1993).

16. Chen, S. Doolen, G. D., Kraichnan, R. H. \& She, Z.-S., Phys. Fluids A 5, 458 (1993).

17. She, Z.-S., Chen, S., Doolen, G., Kraichnan, R. H. \& Orszag, S. A.; Phys. Rev. Lett. 70, 3251 (1993).

18. Zhen-Su She \& Emmanuel Leveque, Phys. Rev. Lett. 72, 336 (1994).

19. Jiménez, J., Wray, A. A., Saffman, P. G. \& Rogallo, R. S., J. Fluid Mech. 255, 65 (1993); also Jiménez, J. \& Wray, A. A., Meccanica, Sept. (1994).

20. Yeung, P. K. \& Brasseur, J. G., Phys. Fluids A 3, 884 (1991).

21. Ruetsch, G. R. \& Maxey, M. R., Phys. Fluids A 4, 2747 (1992).

22. Burgers, J. M., Verhandel. Kon Nederl. Akad. Wetenschappen Amsterdam, Afdeel. Naturkunde 43, pp. $2-12$ (1940); also in Advances in Applied Mechanics 1, 171 (Academic Press, 1948).

23. Yeung, P. K. \& Pope, S. B., J. Fluid Mech. 207, 531 (1989).

24. Squires, K. J. \& Eaton, J. K., Phys. Fluids A 2, 1191 (1990).

25. Elghobashi, S. \& Truesdell, G. C., Phys. Fluids A 5, 1790 (1993).

26. Tio, K.-K., Liñán, A., Lasheras, J. C. \& Gañán, A. M., J. Fluid Mech. 254, 671 (1993).

27. Bray, K. N. C. \& Cant, R. S., Proc. R. Soc. Lond. A 434, 217 (1991). 
28. Markstein, G. H., Nonsteady Flame Propagation, Pergamon Press, Oxford, 1964, p. 8.

29. Osher, S. \& Sethian, J. A., J. Comp. Phys. 79, 12 (1988).

30. Kerstein, A. R., Ashurst, W. T. \& Williams, F. A., Phys. Rev. A 37, 2728 (1988).

31. Peters, N., Premixed, Non-Premixed and Partially Premixed Turbulent Combustion with Fast Chemistry, Proceedings of Joint Meeting of the British and German Sections of The Combustion Insititute, Cambridge, March 1993.

32. Ashurst \& Shepherd, Comb. Sci. \& Tech., to be submitted (1994).

33. Ashurst, Wm. T., Constant-Density Markstein Flamelet in Navier-Stokes Turbulence, Comb. Sci. \& Tech., in press (1994).

34. Murray, J. D., Mathematical Biology, p. 282 (Springer-Verlag, 1989).

35. Collins, L. R., Spectral Analysis of a Simulated Premixed Flame Surface in Two Dimensions, submitted to Comb. Sci. \& Tech. Sept. 1993.

36. Peters, N., J. Fluid Mech. 242, 611 (1992)

37. Wirth, M. \& Peters, N., Twenty-Fourth Symposium (International) on Combustion, The Combustion Institute, 493 (1992).

38. Zhu, J. \& Ronney, P. D., Simulation of Front Propagation at Large Non-dimensional Flow Disturbance Intensities. Comb. Sci. \& Tech., in press (1994).

39. Bradley, D., Twenty-Fourth Symposium (International) on Combustion/The Combustion Institute, 247 (1992).

40. Shepherd, I. G., Some Effects of Heat Release in Premixed Flames, Western States, March 1994; and Shepherd, I. G. \& Kostiuk, L. W., Comb. \& Flame 96, 371 (1994).

41. Gonralez, M., Borghi, R. \& Saouab, A., Comb. \& Flame 88, 201 (1992).

42. Duclos, J. M., Veynante, D. \& Poinsot, T., Comb. \& Flame 95, 101 (1993).

43. Chin, Y.-W., Matthews, R. D., Nichols, S. P. \& Kiehne, T. M., Combust. Sci. \& Tech. 86, 1 (1992).

44. Bielert, U., Klug, M. \& Adomeit, G., SAE 940211.

45. Boulouchos, K., Steiner, T. \& Dimopoulos, P., SAE 940476.

46. Checkel, M. D. \& Thomas, A., Comb. \& Flame 96, 351 (1994).

47. Ting, S.-K., Checkel, M. D., Haley, R. \& Smy, P. R., SAE 940687. Flame radii up to $84 \mathrm{~mm}$ are in: Palm-Leis, A. \& Strehlow, R. A. Comb. \& Flame 13, 111 (1969).

48. Ashurst, Wm. T., Checkel, M. D., \& Ting, D. S.-K., The Eddy Structure Model of Turbulent Flamelet Propagation, the Expanding Spherical and Steady Planar Cases. Comb. Sci. \& Tech. in press (1994).

49. Kwon, S., Wu, M.-S., Driscoll, J. F. \& Faeth, G. M. Comb. \& Flame 88, 221 (1992).

50. Gülder, Ö. L., Twenty-Third Symposium (International) on Combustion/The Combustion Institute, 743 (1991).

51. Liu, Y. \& Lenze, B., Twenty-Second Symposium (International) on Combustion/The Combustion Institute, 747 (1988).

52. Ashurst, Wm. T., Comb. Sci. \& Tech. 92, 87 (1993).

53. Kosiuk, L. W. \& Bray, K. N. C., Comb. Sci. \& Tech. 95, 193 (1994).

54. Lee, T.-W., Lee, J. G., Nye, D. A. \& Santavicca, D. A., Comb. \& Flame 94, 146 (1993).

55. Samaniego, J.-M., Stretch-induced quenching in flame-vortex interactions, Center for Turbulence Research, Annual Research Briefs 205 (1993).

56. Roberts, W. L., Driscoll, J. F., Drake, M. C. \& Goss, L. P. Comb. \& Flame 94, 58 (1993).

57. Baum, M., Poinsot, T. J., Haworth, D. C. \& Darabiha, N., Using Direct Numerical Simulation to Sturdy $\mathrm{H}_{2} / \mathrm{O}_{2} / \mathrm{N}_{2}$ Flames with Complex Chemistry in Turbulent Flows, submitted to J. Fluid Mech. (1994).

58. Poinsot, T. J., The Combustion Program at CTR, Center for Turbulence Research, Annual Reseanch Briefs 195 (1993). 


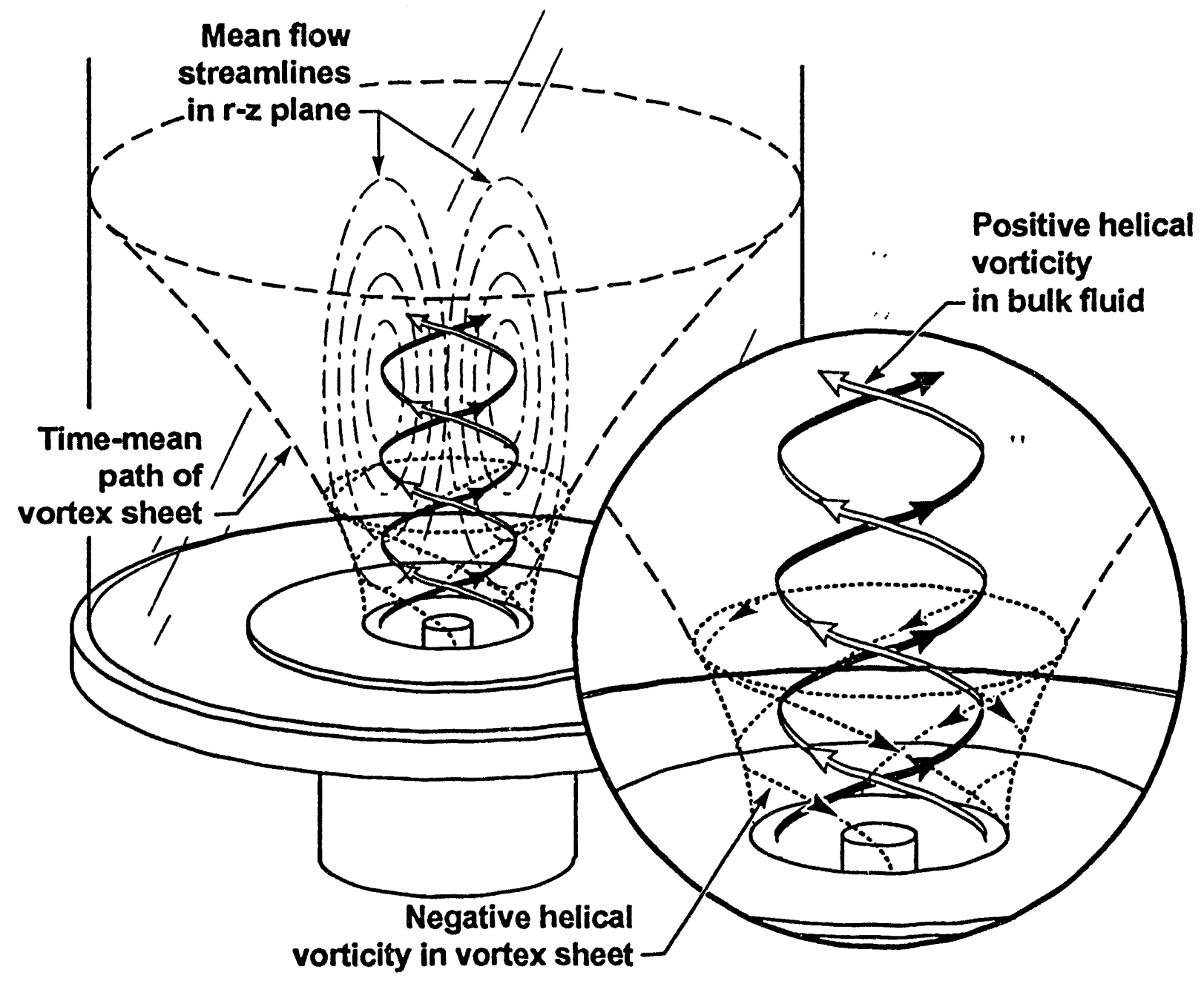

Figure 1. Sketch of swirling vortex structure, the vorticity lines are wound in a left-hand helix which creates a right-hand flow rotation. This unsteady Lagrangian structure convects spray droplets to different locations depending upon droplet size and instantaneous location of the vorticity and this dispersion pattern does not correlate very well with the time-average axisymmetric flow pattern. 


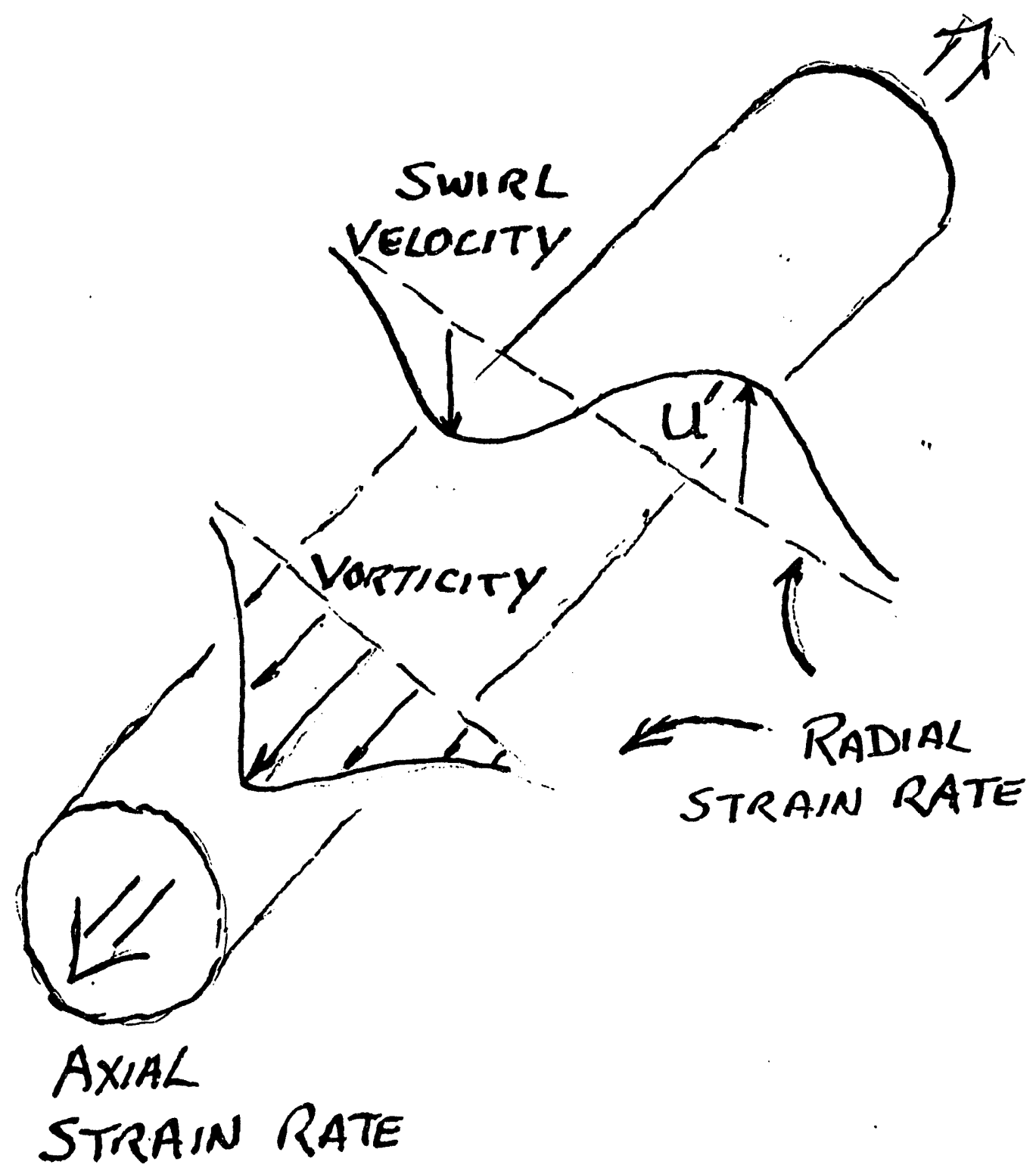

Figure 2. In a Burgers' vortex the axial and radial strain rates maintain a Gaussian vorticity distribution; from the simulations the $1 / \mathrm{e}$ radius is $4 \eta$ and the circulation is $22 \nu \sqrt{R e_{\lambda}}$ which gives a maximum swirling velocity of $u^{\prime}$. Passive propagation normal to the vortex axis is shown in Figure 3. 

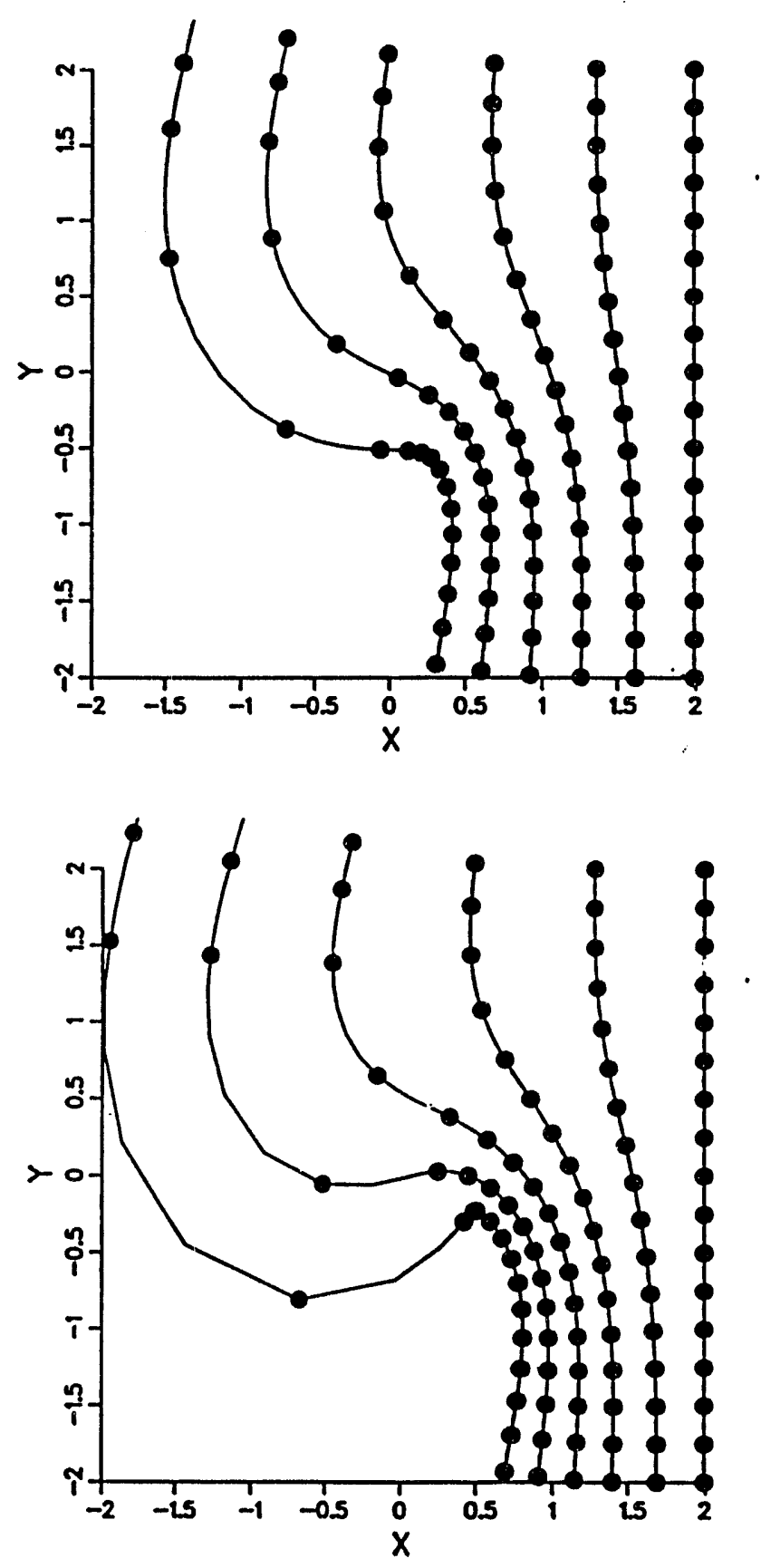

Figure 3. Passive propagation in the swirling flow shown in Figure $2, u_{\theta}=(7 / 4)\left(u^{\prime} / r\right)[1-$ $\left.\exp \left(-r^{2}\right)\right]$ where the radial distance is in units of $4 \eta$. The time interval between images is $2 \eta / S_{L}$. The filled circles are points which are moved in the direction normal to the local flame surface and the change in their spacing indicates the flame stretch (excessive positive stretch also shows the segments used to define the motion). In a) $u^{\prime}=0.5 S_{L}$; the sixth image shows a compression region, but with $u_{\theta}<S_{L}$ there is no cusp at this time. In b) $u^{\prime}=0.8 S_{L}$; the sixth image does show a cusp formation. From passive turbulence simulations, approximately $30 \%$ of the flame surface has compressive strain. Generation of flame area may exhibit a different functional behavior when cusp formation is possible and so extrapolation of $S_{T}$ versus $u^{\prime}$ from $u^{\prime}=S_{L}$ towards $u^{\prime}$ of zero may require different functions. These images neglect the radial strain rate; to include it would require specification of $R e_{\lambda}$ (in order to define $\lambda$ compared to $\eta$ ). 
Strain Rate $\quad \alpha>\beta>\gamma$

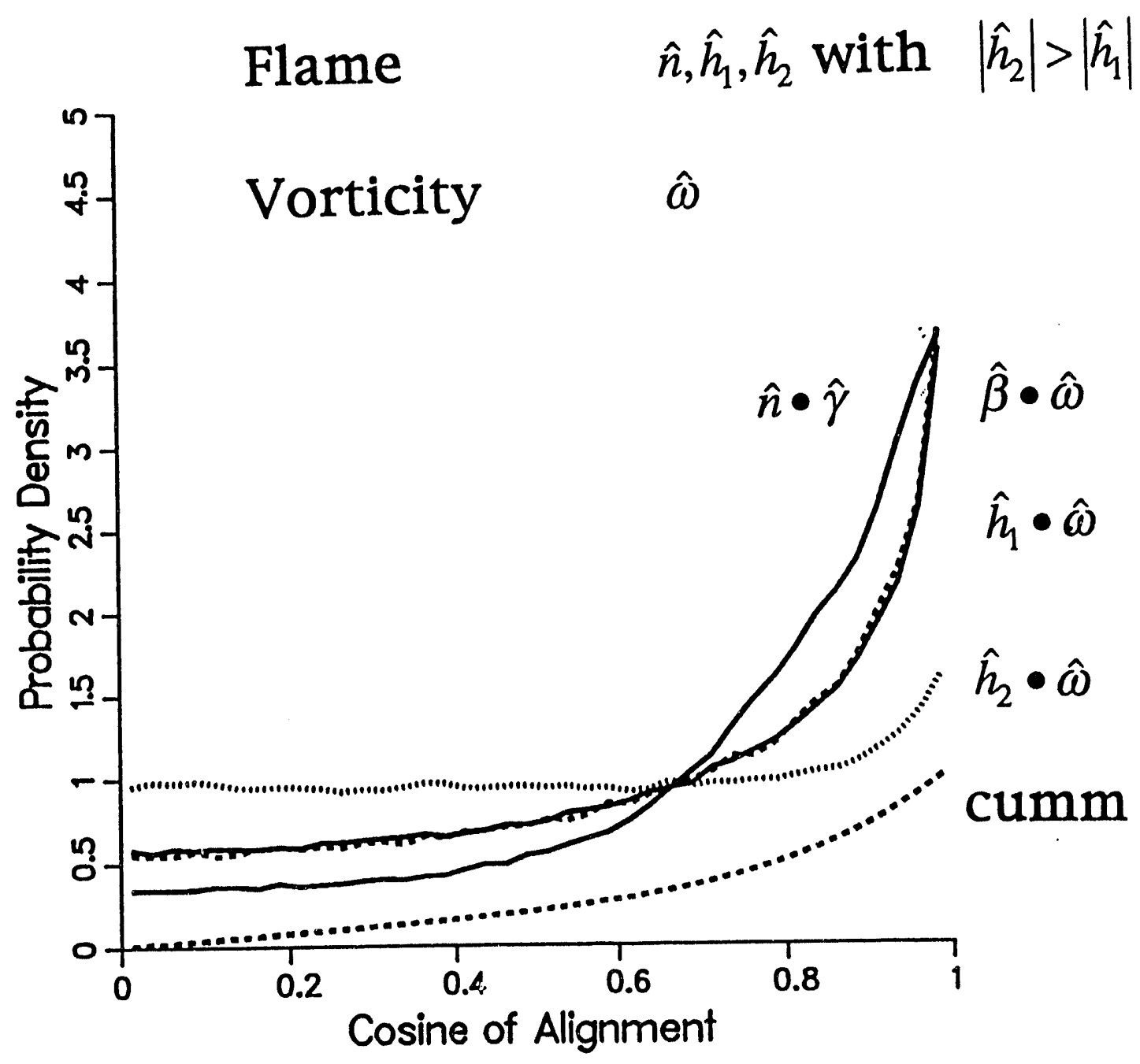

Figure 4. Flame surface alignment with the compressive strain rate is given by $\mathbf{n} \cdot \gamma$, the cummulative probability of this distribution shows that more than half of the flame surface is within 37 degrees of the $\gamma$ direction. The intermediate strain rate $\beta$ and the smallest curvature eigenvalue $h_{1}$, align with the vorticity direction which is consistent with the large probability for a cylindrical flame shape. 

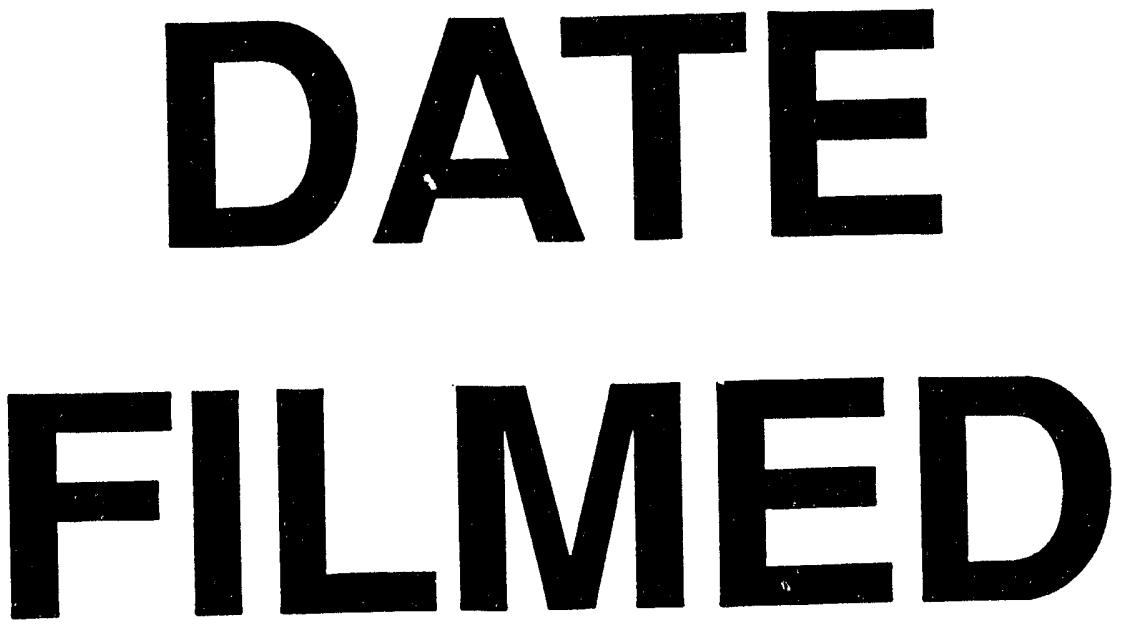

$9 / 12 / 94$
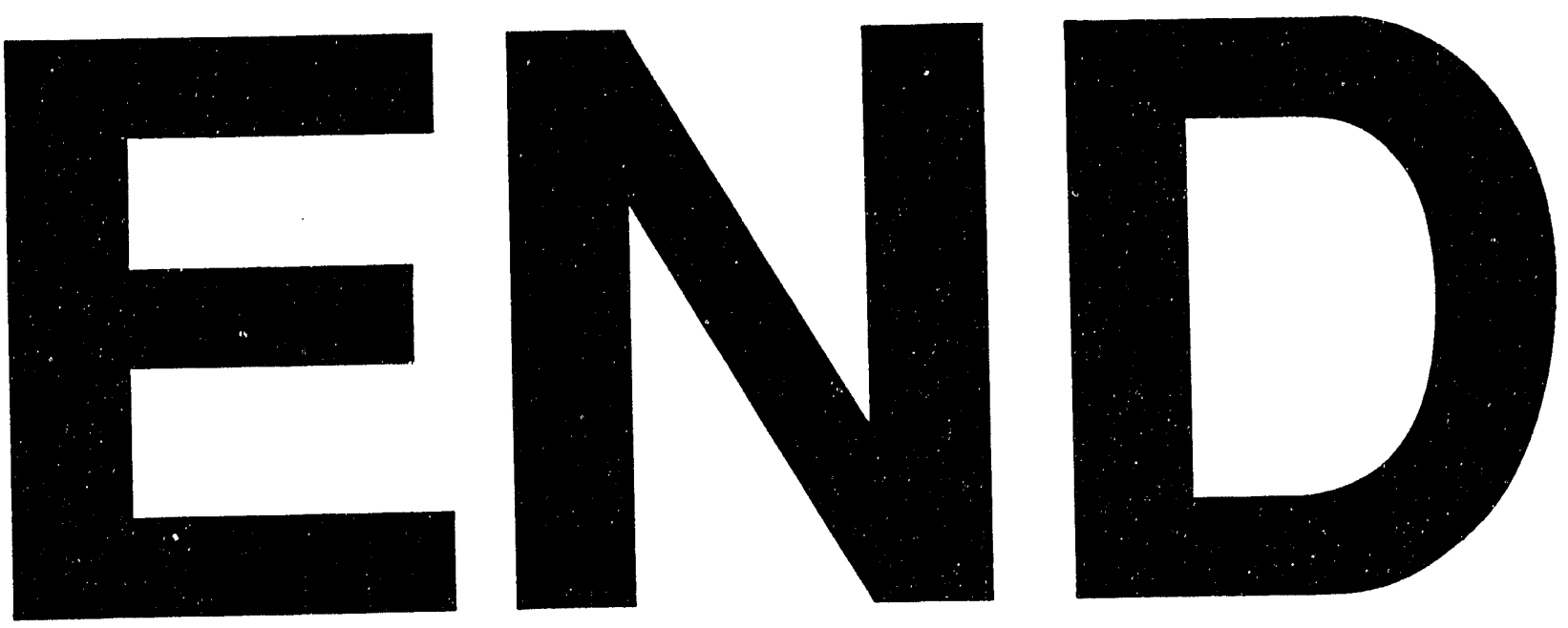
$\longrightarrow$ 